\title{
Die Theorie gesellschaftlicher Modernisierung und das Problem gesellschaftlicher Integration ${ }^{1}$
}

\author{
Ditmar Brock \\ Matthias Junge
}

Technische Universität Chemnitz-Zwickau, Philosophische Fakultät, PF 964, D-09107 Chemnitz

Zusammenfassung: Die Theorie funktionaler Differenzierung beherrscht das gegenwärtige soziologische Denken zum Themenkreis gesellschaftliche Modernisierung. An ihr entzündet sich insofern jedoch ein zunehmendes Unbehagen als sie eindeutig zu kurz greift, wenn man sie als Gesamttheorie gesellschaftlicher Modernisierung interpretiert. Deswegen häufen sich auch die Versuche, ihren Bedeutungsgehalt zu präzisieren und zugleich vernachlässigte Aspekte zu thematisieren. Unseres Erachtens gehen diese Versuche jedoch noch nicht weit genug. Deswegen wird in diesem Beitrag eine Ergänzung des Theorems funktionaler Differenzierung um einen weiteren Themenkomplex vorgeschlagen, der in dem soziologischen und sozialphilosophischen Denken zum Thema gesellschaftliche Modernisierung immer schon eine wichtige Bedeutung hatte. Bisher wurde er jedoch eher als Alternative gegenüber der Theorie funktionaler Differenzierung verstanden. Das soziologische Verständnis gesellschaftlicher Modernisierung soll dadurch weiterentwickelt werden, daß das Theorem funktionaler Differenzierung mit dem im Liberalismus und Marxismus betonten Modell der zunehmenden Vergesellschaftung über den Markt zusammengeführt wird.

\section{Einleitung}

Man hat der Soziologie immer wieder vorgeworfen, daß sie sich in den Elfenbeinturm ihrer Wissenschaftlichkeit zurückziehe (z.B. Beck 1980). Was den Praxisbezug und die Neigung anbelangt, konkrete Empfehlungen auszusprechen, ist dieser Vorwurf sicher nicht aus der Luft gegriffen. In einer anderen Hinsicht hat die Soziologie jedoch immer Offenheit, oft eine nur allzugroße Offenheit bewiesen. Die zentralen Begriffe und Konzepte, mit denen sie den Themenkreis Modernisierung und moderne Gesellschaften bearbeitet, lassen unschwer den Einfluß des jeweiligen "Zeitgeistes" erkennen. Um im Bilde zu bleiben: Nach dieser Seite hin waren die Fenster und Türen des soziologischen Elfenbeinturmes schon immer sperrangelweit offen. Solche Einflüsse sind so lange überhaupt kein Problem, als sie nicht zu panikartigen Absetzbewegungen und vorschnellen Kehrtwendungen verleiten. Aber gerade dieses ist derzeit zu. beobachten. Die heiße Kartoffel, die viele derzeit nur allzuschnell loswerden wollen, heißt Fortschrittsoptimismus oder vorsichtiger formuliert: evolutionstheoretische Annahmen. Ein Gestus abgeklärter Skepsis gegenüber Fortschrittshypothesen jeglicher Art ist in den letzten Jahren wieder in Mode gekommen (vgl. z. B. Hondrich 1992).

'Für Hinweise und Hilfestellung möchten wir uns bei Hans Joas bedanken.
Auf der viel beachteten Tagung der Sektion Soziologische Theorie zum Thema "Modernität und Barbarei“, saßen Arnold Gehlen und Hans Freyer gewissermaßen unerkannt mit auf dem Podium. Dies ist symptomatisch. Obwohl vieles von dem, was in Hamburg vorgetragen wurde, unausgesprochen an die ,Theorie des gegenwärtigen Zeitalters" anknüpfte, wurde dies nicht wahrgenommen. Wie so vieles andere wurde auch Freyers Theorie sekundärer Systeme bei der vorletzten Kehrtwende soziologischen Denkens Mitte und Ende der 60er Jahre auf den Kehrichthaufen der Geschichte geworfen mit der Folge, daß diese wie auch viele andere Überlegungen nicht weiter tradiert wurden und heute zum Teil auf Umwegen wieder neu gewonnen werden müssen. ${ }^{2}$ Der Zeitgeist hat damals so kräftig durch den soziologischen Elfenbeinturm geweht, daß der Soziologie zahlreiche wichtige Konzepte und Gedanken abhanden gekommen sind. Auf ähnliche Weise wie in der zweiten Hälfte der 60er Jahre modernisierungskritische Überlegungen der Soziologie ,abhanden" gekommen sind, so droht heute allen Spielarten evolutionstheoretischen Denkens ${ }^{3}$ dasselbe Schicksal.

Vor diesem Hintergrund möchten wir uns vom Wehen des Zeitgeistes nicht zu unüberlegten $\mathrm{Ab}$ setzbewegungen verleiten lassen, sondern in die-

\footnotetext{
${ }^{2}$ Vgl. hierzu nur die Rekonstruktion der Arbeiten Freyers, von Martins und Heimanns durch Kruse (1994).

${ }^{3}$ Vgl. etwa den Reader von Müller/Schmid (Hrsg.) 1994, der fast ausschließlich handlungstheoretische Ansätze einer Theorie des sozialen Wandels umfaßt.
} 
sem Beitrag etwas anderes versuchen. Wir möchten die These vertreten, daß die konzeptionellen Probleme im Verhältnis von gesellschaftlicher Modernisierung und sozialer Strukturbildung sehr viel damit zu tun haben, daß sich die Soziologie den Phänomenen gesellschaftlicher Modernisierung unter drei voneinander isolierten und weitgehend gegeneinander abgeschotteten Argumentationsstrategien nähert. Die erste Argumentationsstrategie ist mit dem Theorem funktionaler Differenzierung (vgl. Wehling 1992) umrissen. Modernisierung erscheint aus dieser Perspektive als immer weiter fortlaufender Proze $B$ der Ausdifferenzierung eigenlogischer spezialisierter Systeme. Der zweite Argumentationsstrang war in Form einer Theorie der Produktivkraftentwicklung bzw. Kapitalismusentwicklung in den $70 \mathrm{er}$ Jahren populär. Heute hat er dagegen seine Bedeutung nahezu vollständig eingebüßt. Wir wollen an diese Denktradition auf eine wesentlich weniger zugespitzte Art und Weise anknüpfen und eine Theorie des Ressourcentransfers formulieren, die die Theorie funktionaler Differenzierung an einigen wichtigen Stellen zu ergänzen vermag. Ein in diesem Beitrag noch ausgesparter dritter Argumentationsstrang ist mit den Begriffen Individualisierung und Zivilisierung umschrieben. Insofern knüpft dieser Beitrag an Autoren an, die sich bemüht haben, die einzelnen Phänomene des gesellschaftlichen Modernisierungsprozesses untereinander zu verknüpfen. Zu nennen sind hier u.a. Max Webers Rationalisierungstheorie (1988) und Norbert Elias' Zivilisierungstheorie (1976).

Auf dem Wege einer Verbindung der drei zentralen Traditionslinien soziologischen Denkens über Modernisierung läßt sich unseres Erachtens ein Theoriegerüst entwickeln, das breit genug und für unterschiedlichste Fragestellungen hinreichend offen ist. ${ }^{4}$ Es könnte die Möglichkeiten der Akkumulation soziologischen Wissens über den Prozeß gesellschaftlicher Modernisierung verbessern und

\footnotetext{
${ }^{4}$ Dieser Zuschnitt der Fragestellung unterscheidet unser theoretisches Vorhaben von einem fruheren Versuch Bergers (1986) in zweierlei Hinsicht: die von Berger idealtypisch vorgenommene Gegenuberstellung von Marxismus und Modernisierungstheorie muß heute ergänzt werden um die Reaktualisierung der mit dem Stichwort Individualisierung angesprochenen Traditionslinie, andernfalls drohen soziologische Debatten um Probleme gesellschaftlicher Integration zu schnell in das Fahrwasser der Postmodernismusdiskussion (Lyotard 1986) zu geraten. Zweitens steht in unserem Beitrag nicht die Frage im Mittelpunkt, ob ein "nachmodernes" Gesellschaftsstadium denkbar ist.
}

damit auch eine gewisse Gewähr dafür bieten, daß nicht eine erneute Drehung des Zeitgeistes zu einem erneuten abrupten Traditionsbruch führt.

Vom Zeitgeist wollen wir uns im folgenden nur insoweit beeindrucken lassen, als auch wir konzedieren, daß der Prozeß gesellschaftlicher Modernisierung immer wieder Probleme gesellschaftlicher Integration aufwirft. Daß dies aber zu einer apodiktischen Lesart gesellschaftlicher Modernisierung derart führen müßte, daß Modernisierung auf zwangsläufige Weise Desintegration hervorbringen müsse, erscheint uns dagegen eher zweifelhaft. Dennoch ist das Problem gesellschaftlicher Integration der zentrale Angelpunkt, an dem die Leistungsfähigkeit jeder soziologischen Theorie gesellschaftlicher Modernisierung abgelesen werden kann. Ein kurzer Blick auf aktuelle Forschungsthemen, in den zeitdiagnostischen Blätterwald oder auch in die von den Demoskopen erhobenen Hitlisten gesellschaftlicher Ängste zeigt uns, wie aktuell dieses Thema gegenwärtig wieder ist.

\section{Die soziologische Tradition und die aktuelle Diskussion}

Der mit der soziologischen Theoriegeschichte vertraute Leser wird unschwer erkennen, daß wir Weichenstellungen in der Theorieentwicklung, die insbesondere mit dem Namen Durkheim verknüpft sind, in gewisser Weise noch einmal nachvollziehen, um Verknüpfungspunkte zwischen diesen drei in ihren Eigenlogiken festgefahrenen Traditionslinien soziologischen Denkens über die Moderne offenzulegen. Der gemeinsame Hintergrund und damit auch das Fundament unserer Uberlegungen ist letztlich die unbestrittene Tatsache, daß Modernisierung mit Arbeitsteiligkeit und den damit zusammenhängenden. Prozessen der Freilegung individueller Potentiale auf der einen und dem Problem der Handlungskoordination und Sozialintegration auf der anderen Seite zu tun hat. ${ }^{5}$

\footnotetext{
${ }^{5}$ Die hier verfolgte Fragestellung verläuft jedoch nicht analog der von Lockwood (1964) vorgenommenen Unterscheidung von Sozial- und Systemintegration, sondern sie zielt auf eine als eigenständige Integrationsform verstandene Verbindung zwischen Sozial- und Systemintegration in Form eines Ressourcentransfers. Eine solche Konstruktion vermeidet sowohl die Diskussion um die MikroMakro-Verknüpfung (vgl. Alexander/Giesen 1987) wie auch unbefriedigende Konzeptionen einer „Umstellung“ der Integrationsform von Handlungsorientierungen auf Handlungsfolgen (etwa Habermas 1981). Vielmehr nimmt
} 
In diesem Abschnitt werden wir zunächst die drei zentralen Traditionslinien rekapitulieren. Unsere Aufmerksamkeit gilt dabei einmal der Frage, wo und wie der entscheidende Strukturbruch zwischen vormoderner und moderner Gesellschaft markiert wird. Zum anderen wollen wir skizzieren, wo die Unvereinbarkeiten und wo die gegenseitigen Blindstellen liegen. Drittens schließlich gilt unser Interesse der Art und Weise, wie jeweils das spezifísche Integrationsproblem moderner Gesellschaften angesetzt wird.

Durkheims Studie über die Arbeitsteilung (1988) steht nicht am Beginn der Theorie funktionaler Differenzierung. Doch kann dieses Werk mit seiner gleichzeitigen Wendung gegen den evolutionären Optimismus und Organizismus eines Spencer ${ }^{6}$ und gegen das utilitaristische und voluntaristische Modell des Gesellschaftsvertrags eines Hobbes oder Smith exemplarisch für die Möglichkeiten und Schwierigkeiten dieses Theoriemodells stehen. Durkheim verneint in seiner Fragestellung nach den Bedingungen gesellschaftlicher Integration den ontologischen Primat des Individuums und ersetzt ihn durch den ontologischen Vorrang der Gesellschaft. Damit stellt sich für ihn die Frage nach der gesellschaftlichen Integration als Frage nach den Bedingungen der Integration von Individuen in eine funktional differenzierte Gesellschaft. Mit der Ausdifferenzierung von Handlungs- und Wissensbereichen infolge einer zunehmenden Spezialisierung der Arbeit $^{7}$ kann, so Durkheim, Integration nur durch den Rückgriff auf die nicht-kontraktuellen Elemente des Vertrages und einen wachsenden Individualismus erklärt werden. Arbeitsteilung erzeugt den Individualismus und dieser wiederum treibt die funktionale Differenzierung voran. Beide Prozesse verstärken

sie eine Frage auf, die bereits Parsons (1980) in seiner Theorie der generalisierten Austauschmedien stellte: Welche Input-Output- Beziehungen regulieren auf welche Weise die Integration zwischen Systemen? Nur werden wir diese Frage hier nicht im Zuge der Entwicklung einer Medientheorie beantworten (zur Kritik vgl. nur Künzler 1989; Willke 1991: 156-181), sondern in Form der Rekonstruktion einer Sphäre des Ressourcentransfers, die sowohl spezialisierte Systeme und den privaten Lebensbereich integriert, wie sie auch selber zum entscheidenden Mechanismus gesellschaftlicher Integration geworden ist.

${ }^{6}$ zur Verwandtschaft der Argumentationen von Durkheim und Spencer vgl. Corning (1982)

${ }^{7}$ zur Doppeldeutigkeit des Begriffs der Arbeitsteilung bei Durkheim, die ihm den Anschluß sowohl an die Differenzierungstheorie Spencers wie auch an das Konzept der Arbeitsteilung bei Smith erlaubt, vgl. Luhmann (1988) sich wechselseitig und sichern langfristig die Stabilität gesellschaftlicher Integration in den beiden Problemfeldern struktureller und sozialer Integration (vgl. Tyrell 1985).

Aber bereits in der zweiten Auflage der „Arbeitsteilung" gesteht sich Durkheim im Vorwort zur Neuauflage die Schwäche dieses optimistischen Modells ein und versucht ihr abzuhelfen, indem er auf die Integrationspotentiale korporatistischer Berufsgruppen verweist und sie in das Modell zu integrieren versucht. Seine Selbstmordstudie (1987) schließlich wendet sich vollständig von der Frage nach den Bedingungen struktureller Integration ab und untersucht nur noch die Möglichkeiten einer Integration auf der Ebene der ,sozialen Bande". Sein Forschungsinteresse entfernt sich endgültig von der Problematik der Eigenlogik funktional ausdifferenzierter Systeme. Diese Schwerpunktverlagerung hat, neben vielen anderen in der Rezeptionsgeschichte erwähnten ${ }^{8}$, einen für unsere Fragestellung bedeutsamen systematischen Grund. Michael Schmid (1989) hat in seiner Rekonstruktion des Zusammenhangs von Arbeitsteilung und Solidarität bei Durkheim überzeugend zeigen können, daß Durkheim durch die Vermengung zweier unterschiedlicher Formen des Solidaritätsbegriffs nicht in der Lage war, den ,marktkapitalistischen Charakter der modernen Industriegüterproduktion" zu verstehen (Schmid 1989: 639), sondern ihn als moralisch regulierten Zusammenhang rekonstruierte. Damit verliert er aber die Einsicht in die Dynamik der expandierenden Kraft einer auf freier Assoziation und Tausch basierenden marktökonomischen Produktion gesellschaftlicher Güter und folgt weiterhin seiner polemischen Rezeption der Idee der Marktökonomie. Der Grund für diese Schwäche der Analyse funktionaler Differenzierung liegt neben dem eben ausgeführten nicht zuletzt darin, daß die Individuen bei Durkheim nicht frei, sondern bis in ihre anthropologischen Grundfesten hinein durch soziale Strukturen bestimmt sind. Bei Durkheim ergibt sich gesellschaftliche Integration als Konsequenz des geradezu totalitären Zugriffs des Sozialen auf die Individuen. Durkheim konnte die Autonomie des Individuums nur unzureichend denken (vgl. Joas 1992: 76 ff).

\footnotetext{
${ }^{8}$ etwa die Kritik von Giddens an Durkheims Funktionalismus (1976), Kritik an der Logik der Modellbildung Durkheims durch Müller/Schmid (1988) oder schließlich die Kritik Tyrells (1985) an der Vermengung der Frage nach struktureller Integration mit der nach den ,sozialen Banden“"
} 
Der entscheidende Strukturbruch zwischen vormoderner und moderner Gesellschaft liegt für Durkheim in einem Umschlagen sozialer Strukturbildung von segmentärer auf funktionale Differenzierung. Gesellschaften entwickeln sich nicht mehr über die Aneinanderreihung ähnlicher und relativ autarker Dorf- bzw. Stadtstrukturen. Für die Entwicklung moderner Gesellschaften ist vielmehr charakteristisch, daß sie in ein Nebeneinander spezialisierter Funktionssysteme zerfallen, zwischen denen eine gegenseitige hohe Abhängigkeit besteht. Im Unterschied zur liberal-marxistischen Traditionslinie koppelt Durkheim sein Verständnis gesellschaftlicher Modernisierung nicht an den im ausgehenden Mittelalter einsetzenden Prozeß $B$ der Erkämpfung politischer und bürgerlicher Freiheitsrechte. Er setzt vielmehr auf eine wesentlich ältere Entwicklung, die bereits den Unterschied zwischen Stammesgesellschaften und den frühen Staatsbildungen ausmacht. Die historische Pointe des Theorems funktionaler Differenzierung ist jedoch, daß diese Tendenz erst mit dem Durchbruch zur modernen Industriegesellschaft wirklich bestimmend wird. Erst dann kann von mehreren funktionsspezifischen gesellschaftlichen Zentren gesprochen werden, deren Autonomie weitere Modernisierungsschritte im Sinne der Verfolgung spezialisierter Sachlogiken sichert. Mit diesem theoretischen Zuschnitt kann Durkheim demgemäß auch nicht sehen, welche wesentlichen Leistungen die Individuen für die Reproduktion und die Fortschreibung sozialer Systeme und sozialer Strukturen leisten.

Die von Durkheim vehement kritisierte Tradition des Liberalismus, die letztlich in die politische Ökonomie von Marx einmündet, stellt im Gegenzug die Bedeutung des Marktes und die Dynamik einer auf Vertrag basierenden Marktökonomie in den Mittelpunkt. Aus diesem Blickwinkel wird der Traditionsbruch zwischen moderner und vormoderner Gesellschaft im Übergang zwischen Mittelalter und Neuzeit lokalisiert. Der Prozeß der Durchsetzung bürgerlicher Freiheitsrechte und der Rationalisierung politischer Herrschaft setzt individuelle Potentiale frei, die auch eine Rationalisierung im Bereich beruflicher Arbeitsteilung erlauben. Charakteristisch in dieser Hinsicht ist sicherlich Adam Smith' berühmtes Beispiel einer manufakturellen Nadelproduktion. Die Überwindung der Grenzen und des Musters handwerklicher Arbeitsteiligkeit durch eine manufakturelle Arbeitszerlegung versprach unerhörte Produktivitätsgewinne und damit auch eine Hebung des allgemeinen Volkswohlstandes. Die von Smith ent- wickelte Denkfigur der ,invisible hand“ wird zur tragenden Säule einer impliziten Theorie gesellschaftlicher Integration. Sie resultiert aus der Assoziation freier Bürger, die in der Verfolgung eigener Interessen eine stabile, expandierende soziale Ordnung herstellen. Aber bereits die Weiterentwicklung des Paradigmas durch den Sozialliberalismus von Mill (1976) oder die politische Ökonomie eines Marx (1973), zeigt auf, daß die unterstellte Annahme einer ,prästabilisierten Harmonie" (Leibniz) der marktkapitalistischen Ökonomie über sich selbst hinaustreibt. Bei Mill muß der Staat in die ökonomische Dynamik eingreifen, um die Expansion des Marktgeschehens unter Kontrolle zu halten. Marx hingegen entwickelt ein Modell der Marktökonomie, das in seiner Evolution die harmonische Ordnung der invisible hand zerstört und die Marktteilnehmer antagonistischen Interessenlagen unterwirft. Die Marktdynamik konstituiert eine eigenständige und ihrer Eigenlogik folgende ökonomische Sphäre, die zur Selbstzerstörung der bürgerlichen Gesellschaft führt. In der Denkfigur einer freien Assoziation der unmittelbaren Produzenten wird dann freilich die Realität wachsender Marktvergesellschaftung zu einem vorübergehenden Zustand erklärt, der überwunden werden muß, um die verlorengegangene soziale Integration wieder zurückzugewinnen. Über Begriffe wie Verdinglichung und Entfremdung unterscheidet Marx zwischen defizitären Formen moderner Vergesellschaftung und einer letztlich in Formen der Vergemeinschaftung wurzelnden ,echten" Sozialintegration (siehe hierzu auch Berger 1986).

Bestimmte Gemeinsamkeiten dieser beiden hier nur kurz skizzierten Theorietraditionen werden in einer dritten Traditionslinie, der Individualisierungsdiskussion, aufgegriffen und zusammengeführt. Bereits am Beginn der Konstitution der Soziologie als einer eigenständigen Wissenschaft steht das Phänomen gesellschaftlicher Individualisierung bei Durkheim (1988) und Georg Simmel (1983; 1992) im Zentrum ihrer Fragestellungen. Suchte Simmel im Individualisierungsprozeß noch nach einem gesellschaftlichen Muster der Integration, so sah Durkheim im Phänomen der Individualisierung im Laufe seiner Werkentwicklung nur noch desintegrierende, zur Anomie führende Tendenzen. Aber auch die Überlegungen Simmels zur Genese von Individualität und Individualisierung bleiben in einer anderen Hinsicht eigenartig zweideutig. Wachsende Individualisierung resultiert aus der „Ausdehnung der Gruppe“ und der im $\mathrm{Zu}$ ge der gesellschaftlichen Entwicklung voranschrei- 
tenden „Kreuzung sozialer Kreise“. Der mit der Individualisierungsthese markierte Traditionsbruch bezieht sich auf einen offenbar für städtische Lebensformen besonders charakteristischen Entmischungsvorgang in den Sozialkontakten. Insbesondere Simmel macht darauf aufmerksam, daß städtische Sozialkontakte aufgabenbezogener geworden sind. In dem Maße, wie sich spezifische Positionen und Rollen ausmachen lassen, die in Interaktionsgeflechten rational verfolgt werden, findet so etwas wie eine Verdichtung in den modernen Sozialkontakten statt. Die unvermeidliche Folge ist, daß sie zugleich damit auch punktueller, strategischer werden und "nicht zugehörige" Aspekte immer weiter ausklammern. Verglichen mit den unspezifischen Interaktionsbeziehungen vor allem dörflicher Gemeinschaften muß daher für moderne Gesellschaften ein je individueller Akteur von seinen aufgabenspezifischen Sozialkontakten abgelöst werden können. Seine Sozialkontakte werden wähl- und gestaltbarer und zugleich der Möglichkeit nach vielfältiger und weitreichender. Dieser Prozeß ist jedoch insofern ambivalent zu bewerten, als dabei zugleich so etwas wie eine stabile soziale Identität verlorengehen muß (Simmel 1983; 267 ff). Unklar bleibt, ob diese Prozesse eine Folge funktionaler Differenzierung oder eine Folge der gesellschaftlichen Durchsetzung der Marktökonomie sind. Diese Unklarheit ist sicherlich bedingt durch den insbesondere in den frühen Arbeiten Simmels noch erkennbaren Einfluß des evolutionstheoretischen Denkens Spencers (vgl. Heckmann/Kröll 1984: 54). Aber diese Ambivalenz hat die Rezeption Simmels daran gehindert, die durch Simmel bereits formulierte (Simmel 1992: 51) Einsicht aufzunehmen, daß das energetische Potential individualisierter Individuen zum entscheidenden Movens der Produktion und Reproduktion sozialer Strukturen wird (siehe hierzu auch Landmann 1987).

Liegt die Schwäche der liberalistischen, aus der schottischen Moralphilosophie erwachsenen Tradition darin, daß sie die Grenzen des Ordnungsmusters der invisible hand nicht sehen kann, so liegt die Schwäche der am Prozeß funktionaler Differenzierung ansetzenden funktionalistischen Tradition darin. daß sie nicht sieht, welche Beiträge Individuen für die Reproduktion und Fortschreibung sozialer Systeme und die funktionale Differenzierung leisten. Die Schwäche der in der Traditionslinie der Individualisierungsdiskussion entwickelten Überlegung sehen wir nicht zuletzt darin, daß sie die im Konzept gesellschaftlicher Individualisierung angelegten Möglichkeiten zur
Analyse der Bedeutung des „menschlichen Potentials" für die sozialen Strukturen nicht nutzt.

\section{Neuere Konzepte zum Verhältnis von Modernisierung und Sozialintegration}

Dieses theoretische Dilemma hat in den letzten Jahren zu vermehrten Anstrengungen geführt, Integration noch einmal neu zu konzipieren. Für sie ist insgesamt charakteristisch, daß das Theorem funktionaler Differenzierung anerkannt und zugleich in unterschiedlicher Weise relativiert wird. $\mathrm{Zu}$ den bekanntesten Versuchen gehören insbesondere die Theorie des kommunikativen Handelns von Jürgen Habermas (1981), die Theorie der Strukturation von Anthony Giddens (1988) und neuere Arbeiten von Hans Joas (1990; 1992), in denen er das Ziel einer nicht funktionalistischen Theorie funktionaler Differenzierung verfolgt. In diesen Zusammenhang gehört weiterhin die Argumentation von Klaus Eder (1988) zum Verhältnis von Poiesis und Praxis, die er im Zuge seiner Überlegungen zur Vergesellschaftung der Natur aufgestellt hat. Auch das wieder erwachte Interesse an der Praxisphilosophie eines Alain Touraine (1976; 1988) oder eines Cornelius Castoriadis (1984) ebenso wie das steigende Interesse an dem dauerhaften Versuch von Richard Münch (1984; 1988; 1994), eine Theorie der Interpenetration sozialer Systeme zu entwickeln - sie alle weisen in dieselbe Richtung.

Habermas (1981) versucht in seiner Theorie des kommunikativen Handelns eine schlüssige Verbindung zwischen den beiden Bereichen der Lebenswelt und des Systems herzustellen, die in diesem Kontext für die beiden Sphären funktional ausdifferenzierter Systeme und der Sphäre der Handlungskapazitäten autonomer Individuen stehen. Daszentrale theoretische Argument von Habermas für eine zweistufige Gesellschaftkonzeption ist, daß die materielle Reproduktion der Lebenswelt niemals das gewollte Resultat gemeinsamer Arbeit sein kann, sondern nur über die Vernetzung funktionaler Folgen und Nebenfolgen vollzogen werden kann. Aber diese zweistufige Gesellschaftskonzeption einer Zusammenführung des handlungstheoretischen Gesellschaftsmodells mit der Perspektive funktionaler Differenzierung scheitert, weil der Status des Übergangs von sozialer Integration zu systemischer Integration empirisch unplausibel und analytisch nicht haltbar ist (vgl. Joas 1986).

Und doch hat die Habermas'sche Argumentation einen wichtigen Aspekt der Verbindung von So- 
zial- und Systemintegration fixiert: Handlungen, die in der Lebenswelt entstehen, gelten als Faktoreingabe für die funktional ausdifferenzierten Systeme. Sie werden dort, und hier machen sich die Anleihen an der Marx'schen Werttheorie bemerkbar, als Realabstraktion behandelt und von ihren kommunikativen Intentionen abgelöst ( $\mathrm{Ha}-$ bermas 1981, Bd.II: 493 ff). Habermas verwendet die Werttheorie von Marx als einen Versuch, um die Übersetzungsregeln im Austausch zwischen System und Lebenswelt zu beschreiben. Die Werttheorie von Marx wird in eine Medientheorie transformiert. ${ }^{9}$ Trotz dieses für den nachfolgend $z u$ entwickelnden Begriff der Ressource und der Sphäre des Ressourcentransfers wichtigen Hinweises, kann der Habermas'sche Versuch der Lösung des Dilemmas zwischen handlungstheoretischer und funktionalistischer, systemtheoretischer Perspektive auf gesellschaftliche Integration im Hinblick auf unsere Fragestellungen nicht befriedigen. Die Eigengesetzlichkeiten funktional ausdifferenzierter Systeme bleiben in der Theorie des kommunikativen Handelns letztlich unzureichend beschriebene Formen, weil die Perspektive von Habermas sehr betont aus der Teilnehmerperspektive entwickelt wird.

Für den Versuch, die Position des Konzepts der funktionalen Differenzierung neu zu bestimmen und dadurch das Dilemma von Liberalismus und Funktionalismus zu lösen, steht sicherlich Richard Münch, der dem ,soziologischen Gemeinplatz“ widersprechen möchte, daß ,die funktionale Differenzierung in relativ unabhängige Systeme und Sphären des Handelns als ein signifikantes Kennzeichen der modernen Gesellschaft zu betrachten“ sei (Münch 1984: 11). Kennzeichen der modernen westlichen Kultur ist vielmehr die Interpenetration, also die gegenseitige Durchdringung funktional differenzierter und rationalisierter Handlungssysteme. Zwischen der Theorie funktionaler Differenzierung und dem Konzept der Interpenetration von Kultur und Gesellschaft ergibt sich dann letztlich eine ähnliche Arbeitsteilung, wie sie Luhmann zwischen Evolutionstheorie und Geschichtswissenschaften ansiedelt (vgl. Luhmann 1975: 159). Funktionale Differenzierung erfaßt die Entwicklungslogik moderner Gesellschaften, die Entwicklungsdynamik erschließe sich dagegen aus der Interpenetration von Kultur und Gesellschaft

\footnotetext{
9 Und damit wird zugleich der entscheidende Gewinn der Marx'schen Analysen verspielt, die Konzeption eines sich selbst regulierenden Marktes als eigenständigem Bereich des Sozialen.
}

(Münch 1993, Bd.I: 9). Aber gegen die Theorie der Interpenetration kann der Einwand erhoben werden, daß Interpenetration als Form der kulturellen Durchmischung normativer Muster unterschiedlicher sozialer Systeme ein für die Gegenwart nicht ausreichendes Verständnis der gesellschaftlichen Entwicklung demonstriert.

Münch hat in einem kürzlich erschienenen Aufsatz (1994) nochmals einen Anlauf unternommen, um das Konzept der Interpenetration analytisch weiterzuentwickeln. Am Beispiel der historischen Entwicklung des Zusammenhangs von Ökonomie und Moral versucht er zu zeigen, daß die Entwicklung von Ökonomie und Moral nicht einer jeweils „einspurigen Eigengesetzlichkeit" folgt (388), sondern beide Systeme eine historisch veränderbare "Schnittmenge“ (398) aufweisen, die als wechselseitige „Durchdringung“ (388) zu einer neuen „Systemeinheit" führt, in der ökonomisches Handeln mit moralischen und moralisches Handeln mit ökonomischen Standards derart durchsetzt werden, daß die Zuteilung von Zahlungen und Achtung durch das System der Berufsarbeit miteinander vermittelt werden. Der von Münch hier erneut vorgetragenen Stellungnahme gegen den Primat funktionaler Differenzierung als entscheidenden Modernisierungsmechanismus können wir nur zustimmen. Aber sein Verständnis von Interpenetration als Durchdringung unterschiedlicher Systeme verengt die Frage nach dem „Bauprinzip der Moderne“" (388) auf den prozessualen Aspekt. Die Durchdringung unterschiedlicher Systeme wird bei Münch nur als Prozeßkategorie gefaßt, ohne zu sehen, daß sich die neu entstehende „Systemeinheit" zu einer eigenständigen Strukturdimension moderner Gesellschaften entwickelt hat. Diese Einsicht bleibt Münch unzugänglich, weil sein Begriff der Interpenetration voraussetzt, daß es eine direkte Übersetzbarkeit der spezialisierten Codes von Ökonomie und Moral gibt. Wenn jedoch funktionale Differenzierung mit wachsender Spezialisierung einzelner Systeme verbunden ist, dann sinkt im Zuge fortschreitender Modernisierung genau diese Wahrscheinlichkeit für eine direkte Übersetzbarkeit unterschiedlicher Codes ineinander. U. E. verlangt die Spezialisierung einzelner Systeme von diesen eine Differenzierung von spezialisierter Innen- und generalisierter Außenperspektive. Diese Differenzierung wird institutionalisiert in Form einer von den Systemen unabhängigen Struktur, die spezialisiert ist auf die „Übersetzung" unterschiedlicher Systemanforderungen. Um im Beispiel des Zusammenhangs von Ökonomie und Moral zu bleiben: der moralisch motivier- 
te Protest von Tierschützern gegen die Pelzindustrie erreicht diese eben nur dann, wenn die moralische Mißbilligung (der Entzug von Achtung) in die Sprache des ökonomischen Systems übersetzt wird als entgangener Gewinn (der Entzug von Zahlungen). Das betroffene ökonomische System reagiert gerade nicht auf die moralische Mißbilligung-diese bleibt ihm unverständliches Hintergrundrauschensondern auf den Entzug von Zahlungen, die die Reproduktion des Systems gefährden, weil sie ihm notwendige Ressourcen entziehen.

Und doch deutet Münch mit dem opaken Konzept der Interpenetration eine Form der Überwindung des in Rede stehenden Dilemmas an, weil im Prozeß der Interpenetration gesellschaftliche Teilsysteme miteinander ,interagieren“ und wechselseitig Leistungen austauschen. Daraus resultiert die Stabilisierung dieser Form gesellschaftlicher Integration und es erscheint denkbar, daß Interpenetration nicht nur eine Prozeßkategorie ist, sondern vielmehr auf eine eigenständige gesellschaftliche Vermittlungssphäre verweist.

Für die radikalere Variante einer handlungstheoretischen Reformulierung des Funktionalismus unter impliziter Beibehaltung eines evolutionstheoretischen Blickwinkels steht Anthony Giddens (Giddens 1988: 284 ff, 350 ff). ${ }^{10}$ Seiner Ansicht nach verfehlen funktionalistische Erklärungen den wissenschaftlichen Anspruch, weil sie eine Beziehung behaupten, ohne sie tatsächlich erklären zu können (351). Für Giddens sind funktionalistische Erklärungen handlungstheoretisch reformulierbar, weil zwischen intentionalem Handeln und dem Selbstlauf nichtintendierter Handlungsfolgen strikt unterschieden werden muß. Menschen handeln intentional ohne alle indirekten Folgen ihres Handelns überschauen zu können. Wenn man nun zusätzlich wie Giddens das Konzept der „Dualität von Struktur“ einführt, dann kann man hoffen, auf dieser Grundlage allmählich Anforderungen an eine soziologische Modernisierungstheorie (ebd. 286) einzulösen.

10) Giddens präsentiert sich zwar als expliziter Kritiker evolutionstheoretischer Annahmen (ebda; 292 ff.). Zugleich schlägt er jedoch eine dreigliedrige Klassifikation von Gesellschaftstypen vor (Giddens 1988), die unter dem Aspekt zunehmender und leistungsfähiger werdender Raum-Zeit-Bindungen konzipiert ist. Die Klassengesellschaft gilt dabei als der „erste wirklich globale Typ gesellschaftlicher Organisation" (Giddens 1988; 237). Darüber hinaus bleibt zumindest unklar, ob Giddens nicht der eigenen Kritik an Evolutionstheorien anheim fällt. Dagegen schützt auch seine Beteuerung nicht, daB es sich hier um .kein verkapptes Evolutionsschema“ (ebda. 236) handle.
Dieser Kritik am funktionalistischen Denken ist sicherlich voll und ganz zuzustimmen. Wahrscheinlich läßt sich jedoch kein soziologisch angemessenes Verständnis gesellschaftlicher Entwicklung über eine Theorie erreichen, die die Reproduktion und Veränderung sozialer Strukturen allein mit den Handlungen von Akteuren zu erklären vermag. Zwar kann Giddens im Rahmen der Theorie der Strukturation die Reproduktion (zumindest die identische Fortschreibung bestehender) sozialer Strukturen recht gut erklären. Menschliches Handeln reproduziert soziale Strukturen und wird umgekehrt von sozialen Strukturen geformt. Für diese handlungstheoretische Reformulierung des funktionalistischen Programms zahlt Giddens indessen einen beträchtlichen Preis. Er besteht darin, daß der in der Theorie funktionaler Differenzierung enthaltene Ansatzpunkt für eine Erklärung gesellschaftlicher Modernisierungsprozesse in diesem begrifflichen Gebäude nicht mehr mittransportiert wird. Aspekte gesellschaftlicher Modernisierung behandelt Giddens deswegen eher deskriptiv entlang der Koordinaten von Raum und Zeit. Er macht auf Veränderungen aufmerksam, ohne daß sie im Rahmen seines Ansatzes soziologisch informativ rekonstruierbar wären.

Gemeinsam ist all diesen Argumentationen dreierlei: zum ersten versuchen alle diese theoretisch ambitionierten Vorhaben nicht mehr nur die Entwicklungslogik sozialer Systeme und gesellschaftlicher Differenzierung nachzuzeichnen, sondern sie versuchen vielmehr, die konkrete Entwicklungsdynamik funktional ausdifferenzierter Systeme mit dem Handlungspotential autonomer Individuen so $\mathrm{zu}$ verbinden, daß Verbindungen zwischen der liberalistischen und der funktionalistischen Perspektive sichtbar werden. In diesem Rahmen ist allen Modellen die zweite Annahme gemeinsam, daß gesellschaftliche Systeme zunehmende Möglichkeiten zur Selbstbeeinflussung haben. Diese Selbstbeeinflussung der gesellschaftlichen Entwicklung durch die Gesellschaft kann dabei sowohl von sozialen Systemen wie auch von handlungsfähigen Akteuren und sozialen Bewegungen ausgehen, wobei der derzeitige Diskussionsschwerpunkt auf der letztgenannten Möglichkeit beruht (vgl. Joas 1992; Touraine 1988).

So richtig und wichtig wie diese theoretische Umorientierung zum handlungsfähigen Akteur und zur Beeinflussung gesellschaftlicher Systeme durch die Gesellschaft selber auch ist, so darf sie nicht dazu verführen, daß nun die Eigenlogik sachlogisch ausdifferenzierter sozialer Systeme unthematisiert bleibt. Dies ist jedoch die dritte Gemein- 
samkeit der vorgenannten theoretischen Anstrengungen. Selbst die Demokratisierung der Differenzierungsfrage von Hans Joas, der bisher elaborierteste Versuch sowohl auf grundlagentheoretischer wie auch auf makrosoziologischer Argumentationslinie die gesellschaftlichen Veränderungen zu denken, verfehlt ein angemessenes und nur im Rahmen systemtheoretischer Deutungsmuster mögliches Verständnis der Eigenlogik ausdifferenzierter sozialer Systeme.

\section{Spezialisierte Wissensentwicklung und Ressourcentransfer - zwei Dimensionen gesellschaftlicher Modernisierung}

Eine Theorie gesellschaftlicher Modernisierung von hinreichender Breite kann unseres Erachtens nur entwickelt werden, wenn das Theorem funktionaler Differenzierung mit den Dimensionen der Vergesellschaftung über Märkte oder marktähnliche Prozesse auf eine Weise ergänzt werden kann, die diese beiden Prozesse auf einander beziehbar macht. Um die Verknüpfung dieser beiden Dimensionen gesellschaftlicher Modernisierung genauer erkennen zu können, ist der einfache, bei Max Weber mehrfach dargestellte Fall der Ausdifferenzierung früher Berufe instruktiv. Zentrale gesellschaftliche Handlungsprobleme wurden nach Weber bereits relativ früh dadurch vorangetrieben, $\mathrm{daB}$ aus ihrer Bearbeitung ein „Beruf" gemacht wurde. Denn nur der Beruf ermöglichte ausgewählten Personen eine kontinuierliche und methodische Konzentration auf dieses Handlungsproblem. Hieraus können sich dann immer klarer fixierte Wissensbestände entwickeln, die tradiert und weiterentwickelt werden können. Auf diesem Wege können dann funktional differenzierte und thematisch spezialisierte Systeme entstehen. Eine Entwicklung in diese Richtung ist aber nur dann möglich, wenn die für die methodische Konzentration auf einen bestimmten Themenbereich freigestellten Personen alimentiert werden können. Deswegen wird beispielsweise die schon eindeutig professionalisierte Tätigkeit des Priesters durch folgende Merkmale charakterisiert: „Die Eingestelltheit eines gesonderten Personenkreises auf den regelmäßigen, an bestimmte Normen, Orte und Zeiten gebundenen und auf bestimmte Verbände bezogenen Kultusbetrieb" (Weber 1985: 260; Hervorhebungen im Original).

Vor diesem Hintergrund möchten wir die soziologische Substanz der Theorie funktionaler Differenzierung folgendermaßen charakterisieren. Der die Evolution bewirkende Mechanismus liegt in der spezialisierten Wissensentwicklung. ${ }^{11}$ Durch eine immer weitere Ausdifferenzierung von Frage-, Problemstellungen und Spezialsprachen lassen sich menschliche Energien auf immer engere Problemfelder hin bündeln. Diese Bündelung ist jedoch keineswegs ausschließlich als kognitiver Prozeß aufzufassen. Sie schließt soziale Prozesse der Autonomisierung zwangsläufig mit ein und dies in zweifacher Hinsicht. Einmal im Sinne einer Freisetzung für die Beschäftigung mit spezialisierten Wissenssystemen. Zum anderen im Sinne einer an den immanenten Sachlogiken des jeweiligen Feldes orientierten und gegen externe Einflüsse relativ abgeschotteten Organisation spezialisierter Problembearbeitung. Im Wissen und in der Wissensentwicklung würde nach dieser Leseart so etwas wie eine überindividuelle Sachautorität stekken, aus der sich eben auch Maximen für die soziale Organisation funktional ausdifferenzierter Subsysteme ziehen lassen. Dieses Wissen, das den Klassikern noch selbstverständlich vertraut war (vgl. Tyrell 1978: 176), scheint nun gerade in jenem historischen Moment verloren zu gehen, in dem der Prozeß dezentralisierter, autonomisierter und funktional ausdifferenzierter Wissensentwicklung eine zuvor ungekannte Dynamik und Reichweite entwickelt hat. In der Habermas'schen Diskurstheorie ist dieser Aspekt der Wissensentwicklung noch in der Form präsent, da $\beta$ wir uns am überzeugenderen Argument orientieren wollen und davon das Eingehen motivierter Bindungen abhängig machen. Diese soziale Bindungen stiftende Autorität beziehen Argumente aus ihrem sachlichen, im jeweiligen Stand der Wissensentwicklung verorteten Kontext. Innerhalb der funktional ausdifferenzierten spezialisierten Systeme ergeben sich $\mathrm{Mu}$ ster einer rationalen, der Tendenz nach egalitären Ordnung aus den in dem jeweiligen Gegenstands-

1 Vgl. generell Luhmann (1993). Nico Stehr (1994) hat kürzlich in seinen Analysen zum Konzept der Wissensgesellschaft betont, daß Wissen ...als Fähigkeit zum sozialen Handeln" (520) zu konzeptualisieren und aus der wachsenden Bedeutung wissenschaftlicher Wissensakkumulation für Handlungen der Individuen die steigende Bedeutung des Wissens als Motor gesellschaftlicher Modernisierung zu begreifen ist. Die Grenzen dieser Analysen liegen jedoch darin, daß sie, ähnlich wie die Ansätze von Daniel Bell und Raymond Aron, Wissensgesellschaften letztlich als Wissenschaftsgesellschaften verstanden wissen wollen. Dem gegenüber liegt dem hier vertretenen Ansatz die Annahme zugrunde, daß die Diffusion wissenschaftlichen Wissens nur einen von mehreren Aspekten gesellschaftlicher Modernisierung darstellt. 
bereich selbst enthaltenen Sachgesetzlichkeiten. Hier liegt eine Quelle nach wie vor überindividueller Vergesellschaftung, die sich nicht auf die Ebene subjektiv-rationalen Handelns bringen läßt.

An Webers Verständnis der Ausdifferenzierung früher Berufe wird aber zugleich deutlich, daß der Prozeß funktionaler Differenzierung von Voraussetzungen abhängt, die im Rahmen dieses Theorems nicht mehr diskutiert werden können. Hier liegt auch die eigentliche Ursache dafür, daß die Vertreter des Theorems funktionaler Differenzierung ihren Erklärungsanspruch auf eine Explikation der Logik gesellschaftlicher Entwicklung zuzuschneiden versuchen. Wir sehen hier indessen eine Chance für eine dimensionale Ergänzung des Theorems funktionaler Differenzierung. Verberuflichung im obigen Sinne bedeutet ja nicht nur spezialisierte Wissensentwicklung, sondern schließt zugleich ein soziales Arrangement ein, das Spezialisierung überhaupt erst erlaubt. Wichtig ist hier, daß ein bestimmter Wissensbereich von bestimmten dafür ausgesuchten Personen betrieben wird und, $\mathrm{da} \beta$ sich deren Berufspraxis auf eine angebbare Klientel richtet. Fortschritte in der Wissensentwicklung werden dadurch möglich, daß der ausgesonderte Personenkreis seine Aktivitäten auf das Spezialgebiet konzentrieren kann. Dies ist in dem Maße möglich, wie er von alltäglichen Routinetätigkeiten (etwa landwirtschaftlicher Tätigkeit) entlastet ist und gesellschaftlich für die Tätigkeit alimentiert wird. Diese zentrale Existenzbedingung wird über eine klientenbezogene kontinuierliche Berufspraxis sichergestellt. Sie stellt die „öffentliche" Seite der Berufsausübung dar, bei der der besondere gesellschaftliche Wert des Berufs demonstriert und spezialisiertes Wissen auch klientenbezogen übersetzt werden muß. Die „Aussonderung" von Personen für einen Beruf muß man sich als eine räumliche, soziale und zum Teil auch zeitliche Herauslösung aus der auf alltägliche Routineverrichtungen hin zugeschnittenen Gemeinschaft vorstellen. Wenn man das Ergebnis von Modernisierung ganz allgemein als Ubergang von lokalen Gemeinschaften zu tendenziell globalen, thematisch ausdifferenzierten Wissens- und Tâtigkeitsbereichen charakterisieren kann, dann fällt der Beginndieses Prozesses mit dem der Ausdifferenzierung von Berufen zusammen.

Die hier zu beobachtenden Mechanismen lassen sich auf den Prozeß systemischer Differenzierung übertragen. Spätestens zu dem Zeitpunkt allerdings, an dem sich das Muster funktionaler Differenzierung weitgehend durchgesetzt hat und wir von einer dezentrierten modernen Industriegesell- schaft sprechen können, muß in Rechnung gestellt werden, daß sich die ausdifferenzierten Systeme nicht mehr aus einer weniger modernen, noch weitgehend unspezifischen sozialen Umwelt versorgen können. An die Stelle einer Ungleichheitsbeziehung zwischen modernisierten Spezialisten und weniger modernen Bauern, also der bei Marx als fundamental unterstellten Ungleichheit zwischen Stadt und Land, tritt nun eine gegenseitige Alimentierung über gleichrangige Marktbeziehungen. Aus dem direkten Tausch von spezialisierten Dienstleistungen und Produkten gegen Naturalien entwickelt sich eine immer weiter verflochtene Tauschsphäre. Und gerade dies ist die Voraussetzung für eine weitere Steigerung der Spezifizität. ${ }^{12}$ Das gesellschaftliche Umfeld muß nun in der Lage sein, nicht nur die Ernährung des ausgesonderten Personenkreises zu übernehmen, sondern hochspezifizierte Funktionssysteme mit ebenso spezialisierten Ressourcen zu versorgen. Spezialisierte Arbeitskräfte, High tech Produkte, Informationssysteme, spezialisierte Serviceleistungen usw. sind die Voraussetzung für weitere Spezialisierungsprozesse.

Die in ihrer Sachlogik ausdifferenzierten Systeme sind für ihre Reproduktion somit auf Ressourcen angewiesen, die sie nicht selber herstellen können. Dafür benötigen sie Zugriffschancen auf systemexterne Ressourcen. Zugleich müssen die sozialen Systeme allerdings umgekehrt in der Lage sein, ihre eigenen Leistungen auch als nachfragbare Ressource für andere Systeme bzw. Individuen darzustellen. Soziale Systeme können sich nur in dem Maße reproduzieren, in dem es ihnen gelingt, sich eine systemexterne Nachfrage nach ihren Leistungen zu schaffen und zuglèich Zùgrîff auf systênexterne Ressourcen zu sichern. Damit ist nicht nur gemeint, daß spezialisierte Systeme für einander strukturierte Umwelten darstellen im Sinne des Theorems der Interpenetration oder einem gemeinsamen Kranz an institutionellen Rahmenbedingungen unterliegen. Ressourcenproduktion und Ressourcenzugriff verlangen einen Übersetzungsproze $ß$ systeminterner Codierungen auf die

12 Mit dem Begriff der Steigerung der Spezifizität greifen wir auf ein Denkmodell zurück, das bereits am Verhältnis von Individualisierung und systemischer Differenzierung als einem allgemeinen Steigerungsverhältnis erfolgreich erprobt wurde. In diesem Modell resultiert aus der funktionalen Differenzierung nicht nur eine Steigerung der Wirkungschancen und der Effektivität der Arbeitsweise sozialer Systeme, sondern ihr läuft eine Steigerung individueller Handlungskapazitäten parallel (vgl. di Fabio 1991). 
Bedürfnisse anonymer Nachfrager hin. In jeder allgemein zugänglichen Ressource steckt immer ein gewisser klientenbezogener ÜbersetzungsprozeB.

Der Ressourcentransfer ist ein gegenüber dem Prozeß funktionaler Differenzierung gleichrangiges Organisationsprinzip hochmoderner Gesellschaften. Gerade weil es in Bezug auf die anderen beiden Lebensbereiche lediglich eine dienende Funktion erfüllt, ist es auf allgemeine Zugänglichkeit und damit auf Universalismus hin strukturiert. Das integrative Potential dieses Bereichs hängt gerade damit zusammen, daß er Möglichkeitsstrukturen bereitstellt, die erst nach Selektionsentscheidungen von Personen oder Systemen strukturierend wirken. ${ }^{13}$ Diese Freiwilligkeit sichert Akzeptanz und Lernprozesse nach dem Muster von Selbstsozialisation. Strukturierend wirkt, daß man immer nur bestimmte Möglichkeiten, die zugleich andere Alternativen definitiv ausschließen, für private Zwecke oder im Rahmen spezialisierter Systeme nutzen kann. Das Telefon ermöglicht eine Kommunikation über räumliche Distanzen hinweg, die zugleich durch die technischen Bedingungen auf die verbale Ebene konzentriert ist. Ebenso wirkt ein Verkehrsnetz oder auch das Buchen einer Urlaubsreise strukturierend. Über die Nutzung der uns zugänglichen Ressourcen wird unser Alltag in einer faktisch verbindlichen Weise vorstrukturiert. Man kann hier auch von materieller Kultur (vgl. Brock 1993) sprechen. Das heißt: Wir können auf die Produkte und Dienstleistungen anderer zurückgreifen, bewegen uns in dichtgeknüpften infrastrukturellen Netzen, die uns mit Strom, Information oder Unterhaltung versorgen, die globale Kommunikation oder auch eine schnelle Fortbewegung ermöglichen und vieles andere mehr. Ihre Verbindlichkeit entspringt aus der Effizienz: Wir müssen uns ihrer Modalitäten be-

\footnotetext{
13. In diesem Sinne umfaßt das System des Ressourcentransfers auch die von Renate Mayntz (1993) kürzlich unter dem Etikett „Infrastruktursysteme“ (100) zusammengefaßten großen technische Systeme. Insbesondere der von ihr erwähnte Ermöglichungscharakter deckt sich mit unserer Kennzeichnung der „dienenden“ Funktion des Ressourcentransfers. Allerdings umfaßt der Begriff des Ressourcentransfers mehr als nur große technische Systeme, vielmehr auch die Institution des Lebenslaufs, den Markt, Bürokratien, und daneben auch Kommunikationsund Verkehrssysteme. Der Ressourcentransfer umfaßt nicht nur technische Artefakte, sondern die sozialen Strukturen in ihrer Gesamtheit. Das hatte bereits Freyer in seiner Kennzeichnung sekundärer Systeme (1955: $92 \mathrm{f}$ ) zum Ausdruck gebracht.
}

dienen, um private oder systemische Zwecke überhaupt oder mit einem Minimum an Aufwand realisieren zu können. Die materielle Kultur legt auf technischem Wege fest, wie etwas getan werden muß, und bringt Menschen in bestimmter Weise miteinander in Verbindung. Das „Was“ und „Warum" bleibt offen; es muß von den Individuen hinzugefügt werden, die sich dieser materiellen Kultur aus ganz unterschiedlichen privaten Motiven heraus bedienen oder sie in den spezifischen Kontext von Funktionssystemen integrieren können. ${ }^{14}$

Der Ressourcentransfer löst die Aufgabe, potentielle Ressourcen systemischer Differenzierung aus lokalen Bindungen (bzw. Naturkreisläufen) herauszulösen und sie als potentielle Ressourcen für spezialisierte Funktionssysteme zugänglich zu machen. Ressourcen lassen sich definieren als potentielle Objekte von Praktiken der Aneignung der materiellen Kultur. Beide Begriffe haben keinen ontologischen Status, sondern sind analytische Hilfsmittel zur Rekonstruktion neuer Integrationsformen und neuer Vergesellschaftungsformen in hochmodernen Gesellschaften. ${ }^{15}$ Ressource kann alles werden, was die Welt ist. ${ }^{16}$ Über Ressourcen kann aber nur verfügt werden, wenn Anrechte auf Ressourcen und d.h. Zugang zum Ressourcentransfer vorhanden ist. Diese Einschrän-

${ }^{14}$ Damit verăndert sich zugleich die Bedeutung von Institutionen für die soziale Ordnung. Noch für Gehlen (1956) drücken sich in Institutionen Leitideen aus. Diese Entlastung von der unmittelbaren funktionalen Zwecksetzung führt jedoch dazu, daß Institutionen mit sekundären Zweckbestimmungen belegt werden. Sekundäre Zweckbestimmung bedeutet, da 3 die einzelnen Individuen über Institutionen frei im Rahmen ihres individuellen Bewußtseins und ihrer „dispositiven Freiheiten“ (Brock 1991) verfügen können. Institutionen werden nach Gehlen geöffnet für individuelle $Z$ wecke und subjektive Motive. Institutionen werden zu Instanzen, die durch die handelnden Individuen selber analytisch, kritisch, zweckrational und funktional betrachtet und benutzt werden können. Damit jedoch wird die Kultur „ein Reservoir technischer Möglichkeiten“ (Schelsky 1973: 24).

15 Dieser Ressourcenbegriff ist verwandt mit dem Warenkonzept von Marx, ohne mit dieser Konzeption identisch zu sein, weil der Ressourcenbegriff weitergefaßt ist: Ressourcen sind nicht nur Waren, sondern ebenfalls Motive und Handlungsenergie, die im rückwärtigen privaten Lebensbereich gewonnen werden und als "dispositive" Freiheiten im Ressourcentransfer wirksam werden und ihn mitgestalten.

${ }^{16}$ Und diese Feststellung verweist zugleich auf die impliziten Grenzen der Expansion des Ressourcentransfers, und damit auf Grenzen dieser Form sozialer Integration: Grenzen der ökologischen Belastung. 
kung verweist darauf, daß Ressourcen den Bedingungen der Knappheit unterliegen. Eine weitere analytische Eigenschaft, die sich aus dem Merkmal der Knappheit ergibt, ist, daß es zwischen Ressourcen Substitutionseffekte geben kann.

Spezialisierte Funktionssysteme hängen also vor allem in dreierlei Hinsicht von der Entwicklung ihres gesellschaftlichen Umfeldes ab. ${ }^{17}$ Einmal vermögen sie nicht für Nachwuchs an Akteuren zu sorgen. Biologische Reproduktion, die Sozialisation von allgemeiner gesellschaftlicher Handlungsfähigkeit muß vorausgesetzt werden und kann nur extern erfolgen. Darüber hinaus haben die spezialisierten Funktionssysteme auch die Herstellung hinreichender Motivation nicht voll im Griff. Dies ist zunächst in dem Sinne gemeint, daß Menschen die Mitwirkung in spezialisierten Systemen als ,,attraktiv" für sich empfinden müssen, also individuelle Reproduktionsinteressen mit systemspezifischen in Verbindung bringen müssen.

Zweitens können spezialisierte Funktionssysteme nicht autonom die gesellschaftliche Nachfrage nach ihren Systemleistungen steuern. Sie sind immer von einem über den Markt bzw. den Staat fixierten „Bedarf“" nach systemspezifischen Leistungen abhängig, über den der Zufluß an Geldmitteln reguliert wird, die dann für systemspezifische $\mathrm{Ak}$ tivitäten eingesetzt werden können.

Drittens sind spezialisierte Funktionssysteme gerade nicht autonome Produktionssysteme. So war beispielsweise die Ausweitung der Klientel für Computer über den rein beruflichen Bereich hinaus eine wichtige Voraussetzung für die hochspezialisierte und kostenintensive Entwicklung immer leistungsfähigerer Chips. Umgekehrt hat die Entwicklung immer leistungsfähigerer und billigerer Computer das Spektrum spezialisierter Anwendungsmöglichkeiten drastisch erweitert. ${ }^{18}$ An diesem Beispiel wird deutlich, wie Prozesse funktionaler Differenzierung mit der Entwicklung des Ressourcentransfers zusammenspielen. Es liegt nämlich in der Logik funktionaler Differenzierung, daß spezialisierte Funktionssysteme die gesamte Fülle benötigter Ressourcen und technischer Möglichkeiten nicht selbst produzieren können. Sie sind davon abhängig, daß ihnen immer spezifischere und in diesem Sinne "nuitzlichere“ Ressourcen zugänglich sind. Die internen Entwicklungsmöglichkeiten hängen gerade auch davon $a b$, ob von einer breiten technischen Innova-

\footnotetext{
17 Vgl. hierzu auch Luhmann (1984 Kap. 5).

${ }^{18} \mathrm{Vgl}$. zur empirischen Untersuchung der Computerdiffusion Allerbeck/Hoag (1989).
}

tion (Schlüsseltechnologien, innovative Verdichtungsräume) möglichst zahlreiche und vielfältige Impulse ausgehen, die systemintern nutzbar sind. Dahinter steht allgemein die Abhängigkeit spezialisierter Funktionssysteme von einer Infrastruktur, die benötigte Ressourcen und technische Möglichkeiten systematisch, effizient, schnell und preiswert zugänglich macht. Jede Spezialisierung bedeutet ja immer eine punktuelle Bündelung von Aktivitäten, was zwangsläufig dazu führt, daß auf immer mehr externe Leistungen effizient zurückgegriffen werden muß. In diesem Sinne sind funktionale Differenzierung und wachsende Verfügung über spezialisierte Ressourcen zwei Seiten ein und derselben Medaille.

Die Angewiesenheit spezialisierter Funktionssysteme auf externe Ressourcen, und insbesondere auf hinreichende Motivation, ist eine Folge von funktionaler und sozialer Differenzierung. So geht Georg Simmel in seiner Soziologie (1992) davon aus, daß es drei apriorische Bedingungen der Möglichkeit von Gesellschaft gibt. Von diesen drei Bedingungen ist in unserem Kontext besonders die zweite von Bedeutung: „daß jedes Element einer Gruppe nicht nur Gesellschaftsteil, sondern außerdem noch etwas ist."(51) Wichtig an diesem zweiten Apriori ist, daß Vergesellschaftung nur in dem Maße möglich ist, in dem Energien, Impulse und Motivationen aus dieser gesellschaftsabgewandten Seite zur Verfügung gestellt werden, um in der Gesellschaft zugewandten Seite zum Tragen zu kommen. Diese Gegenüberstellung von $\mathrm{zu}-$ und abgewandt verweist bereits auf die hohe Bedeutung, die ein privater Lebensbereich für die Produktion und Reproduktion der Gesellschaft insgesamt hat. Aber dieses zweite Apriori Simmels muß in einem modernisierungstheoretischen Sinne interpretiert werden, um es für die hier vorgeschlagene Konzeption gesellschaftlicher Modernisierung frucht" bar zu machen. Dieses Apriori formuliert nicht nur erkenntnistheoretische Voraussetzungen von Soziologie, sondern darüber hinaus historische Voraussetzungen der Entstehung moderner Gesellschaften.

Im Bereich des Ressourcentransfers motivieren Individualinteressen zweckrationale Handlungen. Wenn wir eine bestimmte berufliche Leistung verkaufen oder vertreiben, als Konsument auftreten oder wenn wir Bildungs- und Berufswahlentscheidungen treffen, dann müssen wir uns von unseren persönlichen Interessen leiten lassen, die sich aus den Neigungen ergeben, die wir biographisch entwickelt haben, die mit unseren sozialen und persönlichen Bindungen zusammenhängen, von kul- 
turellen Einflüssen, unserem Status usw. abhängig sind.

Wenn wir uns jedoch innerhalb eines funktional ausdifferenzierten Systems bewegen, dann unterwerfen wir uns immer der Disziplin einer sachlich fixierten Autorität. Wir können eine solche Tätigkeit zwar aus den unterschiedlichsten persönlichen Gründen ausüben. Die Frage eines solchen persönlichen Ertrags wird aber an der Außengrenze, über die Frage der Mitgliedschaft und Nicht-Mitgliedschaft geregelt und spielt dann innerhalb eines ausdifferenzierten Funktionssystems keine direkte Rolle mehr. Die Frage des „persönlichen Ertrags“ einer solchen Tätigkeit kann zumindest von der Konstruktion spezialisierter Funktionssysteme her nicht mehr direkt thematisiert werden.

Auf eine wiederum spezifische Weise kommen Individualinteressen im privaten Lebensbereich zur Geltung. Der private Lebensbereich ist jener Bereich, der aus dem Bereich der Erwerbsarbeit ausgegrenzt ist und komplementär dazu der Regeneration dienen soll. Diese Aufgabenzuweisung verlangt, daß hier die Neigungen und Interessen der konkreten Person thematisch werden und da $\beta$ sie den Bezugspunkt der privaten Aktivitäten abgeben. Im Unterschied zum Bereich des Ressourcentransfers können sie hier also nicht zweckrational verfolgt werden. Sie müssen direkt im Mittelpunkt des Handelns stehen. Es geht also um persönliche Offenheit und um nichts anderes. Der private Lebensbereich ist nicht der Bereich ritualisierten Rollenspiels, sondern wir befinden uns hier hinter der Bühne, im nicht-öffentlichen Bereich (vgl. hierzu nur Simmel (1992) und Goffman (1983)).

Die Abhängigkeit spezialisierter Funktionssysteme vom Ressourcentransfer wird mit dem Begriff der Interpenetration allenfalls ansatzweise erfaßt, jedoch nicht auf den Punkt gebracht. Die von Parsons, Luhmann und Münch vorgelegten Versionen übersehen, daß Systeme in der Regel nicht direkt voneinander profitieren können, sondern erst nach einem Übersetzungsproze $B$ systeminterner Codierungen auf die Bedürfnisse anonymer Nachfrager hin. Ressourcen stellen Leistungen spezialisierter Funktionssysteme dar, die genau einem derartigen Verallgemeinerungsproze $\beta$ unterlagen. Ressourcen bezeichnen gewissermaßen die AuBenseite spezialisierter Funktionssysteme, die für einen Zugriff anderer Systeme entsprechend präpariert sind. Allgemein verständlich präsentierte wissenschaftliche Ergebnisse, ein Brötchen, ein Automobil, ein Film, das Beratungsangebot einer
Behörde - all dies hat Ressourcencharakter. Aus der Perspektive des Ressourcen anbietenden Systems hängt die eigene Reproduktion davon ab, daß diese Ressourcen möglichst häufig genutzt und ihr Nutzeffekt möglichst hoch bewertet wird. Dies kann am einfachsten, aber nicht in jedem Fall, monetär gemessen werden. Aber auch in anderer Form, wie z. B. über wissenschaftliches Renommee kann die Zukunft spezialisierter Funktionssysteme gesichert werden, was nichts anderes bedeutet, als daß ihnen in hinreichendem Maße nun ihrerseits Ressourcen zufließen können. Aus der Nachfragerperspektive sind Ressourcen Produkte spezialisierter Tätigkeit, die einen erkennbaren Nutzen, einen Gebrauchswert ${ }^{19}$ aufweisen.

Ressourcen sollen nicht nur nützlich sein. Sie müssen auch zugänglich sein, um überhaupt genutzt werden zu können. Auch in dieser Hinsicht sind „Übersetzungsleistungen“" gegenüber den zum Teil ja räumlich sehr konzentrierten Funktionssystemen erforderlich. Die räumliche Zugänglichmachung von Ressourcen durch Verkehrssysteme, Massenkommunikation und Nachrichtentechnik ist deswegen auch die wohl zentrale Entwicklung für eine immer weitergehendere und spezialisiertere Ressourcennutzung. Auf dieser Grundlage sind dann weitere Ausdifferenzierungsprozesse möglich.

Die vielleicht naheliegende Vermutung, da $ß$ der alte Marx dasselbe, nur sehr viel präziser vor mehr als 100 Jahren behauptet habe, ist nicht richtig. Für das Verständnis der heutigen, nicht mehr von der Industrieproduktion, sondern zunehmend von sekundären Dienstleistungen geprägten Industriegesellschaften ist es vielmehr notwendig, die für die politische Ökonomie bei Marx entscheidenden Zuspitzungen wieder zurückzunehmen. So mag es für die Analyse des klassischen Kapitalismus hilfreich gewesen zu sein, den Gebrauchswert einer Ware als eine Art notwendiges Vehikel dafür anzusehen, daß sie Träger des sehr viel wichtigeren Tauschwertes werden könne. In einer Zeit, wo wirtschaftliche Wertschöpfung ganz zentral von Innovationen und innovativen Verknüpfungen spezialisierten Wissens abhängt (Reich 1993), wird

19 "Wer durch sein Produkt sein eigenes Bedürfnis befriedigt, schafft zwar Gebrauchswert, aber nicht Ware. Um Ware zu produzieren, muß er nicht nur Gebrauchswert produzieren, sondern Gebrauchswert für andere, gesellschaftlichen Gebrauchswert. ... um Ware zu werden, muß das Produkte dem anderen, dem es als Gebrauchswert dient, durch den Austausch übertragen werden." (Marx $1973 ; 55$ ) 
diese Engführung problematisch ${ }^{20}$. In jedem Falle werden dabei wesentliche gesellschaftliche Verflechtungen ausgeblendet. Ähnliches gilt für den Begriff der Zirkulation, der bei Marx wiederum nur eine Art Vehikel für die Realisierung von Tauschwerten, Profiten und Mehrwert darstellt. Aus heutiger Sicht ist dagegen soziologisch wesentlich folgenreicher, daß die explosionsartige Entwicklung der Verkehrs- und Kommunikationssysteme die Arbeitswelt wie auch den privaten Lebensbereich auf entscheidende Weise umgekrempelt hat. In beiden Lebensbereichen geht es nicht mehr ausschließlich, ja vielleicht nicht einmal primär darum, innerhalb eines gegebenen Rahmens zu arbeiten. Immer bedeutender wird daneben die Selektion aus einem Universum zugänglicher Ressourcen. Berufs- und Reproduktionsarbeit wird auf diese Weise zu etwas, das in den Ressourcenkonsum mit eingebaut ist bzw. diesem punktuell hinzugefügt wird. Dabei darf auch nicht übersehen werden, daß das „Zugänglichmachen" zu einem immer wichtigeren Arbeitsfeld geworden ist, über das ganze gewerbliche Bereiche charakterisiert werden können, wie z.B.: Energieversorgung, Handel, Fremdenverkehr, Automobilwirtschaft, Medien usw. ${ }^{21}$

Entgegen dem bei Luhmann vertretenen Konzept einer offenen und kontingenten Systemumwelt gehen wir davon aus, daß spezialisierte Funktionssysteme sich in einer unter dem Aspekt der Zugänglichmachung strukturierten Umwelt bewegen. Das schließt nicht aus, daß es darüber hinaus unstrukturierte Umweltsegmente gibt. Moderne Gesell-

\footnotetext{
20) Auch für Marx ist Gebrauchswert die fundamentalere Kategorie für die Distribution von Waren. Die Dominanz des Tauschwertes beschreibt für ihn nur die kapitalistischen Verhältnisse. In der hochautomatisierten Produktion sieht Marx Grenzen der Quantifizierbarkeit menschlicher Arbeitsleistungen und damit auch Grenzen des Tauschwertaspektes (vgl. insbesondere Marx 1974; 592 ff). Ein derartiger Entwicklungsgrad der Produktion ist für Marx jedoch nicht unter kapitalistischen, sondern nur unter sozialistisch-kommunistischen Bedingungen denkbar, deshalb zieht er aus diesen Überlegungen auch keine Rückschlüsse auf die eigene Werttheorie.

${ }^{21}$ Eine Ahnung von diesem Zusammenhang muß bereits Jean Fourastié (1954) gehabt haben, als er die gesellschaftlichen Entwicklungen durch Verschiebungen der gesellschaftlichen Produktion vom primären zum sekundären und schließlich zum tertiären Sektor hin gekennzeichnet hat. Dienstleistungen und ihre Zunahme stellen nichts anderes dar, als die zunehmende Notwendigkeit der Ressourcenvermittlung zwischen den ausdifferenzierten Bereichen einer privaten Lebenssphäre und funktional differenzierten sozialen Systemen.
}

schaften zerfallen deswegen nicht in einen Dschungel autopoietisch organisierter Systeme, weil und insofern sie sich in einer unter dem Aspekt der Zugänglichmachung von Ressourcen strukturierten Umwelt bewegen. Hier liegt das gemeinsame gesellschaftliche Band, das auch die individuellen Akteure umgreift.

Wenn diese Überlegungen stichhaltig sind, dann verbietet es sich, eine eindimensionale Logik gesellschaftlicher Modernisierung anzunehmen. Neben der „Differenzierungs- und Spezifizierungslogik" weist gesellschaftliche Modernisierung ebenso eine universalistische Tendenz der Zugänglichmachung von Systemleistungen in Form von Ressourcen auf. Sie führt zu einer immer intensiveren Verknüpfung gerade der hochspezialisierten Systeme über die Nutzung zugänglicher Ressourcen. Während die Entwicklung systematischen Wissens zur Spezifizierung und Ausdifferenzierung zwingt, entwickelt sich daneben ein universalistisch und egalitär strukturierter Bereich des Ressourcentransfers und des Ressourcenaustauschs. Wenn man die freilich problematische Analogie zwischen Gesellschaften und Organismen strapazieren möchte, dann kann man auch davon sprechen, daß die spezialisierten Organe einer Versorgung über Blutbahnen bedürfen. Sie ist ihnen gemeinsam und hält sie funktionsfähig.

Wenn diese Überlegungen zutreffen, dann können wir Modernisierung zunächst als Wechselwirkungszusammenhang zwischen den Entwicklungen von Differenzierung und Universalisierung verstehen. Dieses gegenüber der Theorie funktionaler Differenzierung unseres Erachtens entscheidend erweiterte Verständnis gesellschaftlicher Modernisierung möchten wir im folgenden an drei Beispielen demonstrieren.

Das erste Beispiel handelt vom politischen System. Der Einfluß der Medien auf die Darstellung der Politik, aber auch auf politische Entscheidungen und ihr "timing“, verweist auf die Abhängigkeiten des politischen Systems vom Ressourcentransfer. Im Vergleich zu den klassischen politischen Öffentlichkeiten bietet das Medienzeitalter den Politikern sicherlich ganz wesentlich erweiterte Darstellungsmöglichkeiten gegenüber den Wählern. Dies muß nun aber keineswegs bedeuten, daß der heutige Fernsehzuschauer über politische Vorgänge wesentlich besser informiert wird als die Bürger im Vor-Medienzeitalter. Unlängst hat eine Expertenkommission festgestellt, daß gerade der Kampf der Fernsehanstalten um Einschaltquoten und Marktanteile einen unheilvollen Einfluß auf die 
Darstellung der Politik habe. Damit ist unter anderem gemeint, daß Politik den Bedingungen der Medien und dem Unterhaltungsbedürfnis der $\mathrm{Zu}$ schauer unterworfen wird. An die Stelle von Inhalten würden Personen treten, die das Geschäft der Inszenierung von Politik eben mehr oder weniger gut beherrschen (Hitzler 1994). Die Hintergrundinformation über wichtige Entwicklungen fällt den im Medium Fernsehen besser präsentierbaren „Ereignissen“ zum Opfer usw. Mit anderen Worten: Die Medien verbessern die Möglichkeiten für "Außendarstellungen" der Politik beim Wähler. Dabei wird aber auch deutlich, daß es hier keineswegs um das politische Geschehen selbst geht, sondern um seine medienwirksame Inszenierung. Medienwirksam bedeutet hier, daß Politik ihrerseits zu einer Ressource für den nach Unterhaltung suchenden Fernsehzuschauer wird und dabei mit anderen Sparten des Unterhaltungsmarktes konkurriert. Gegenüber der Darstellung und Inszenierung von Politik muß die Binnenperspektive des politischen Systems strikt unterschieden werden. Zwischen dem innersystemischen Prozeß und der Außendarstellung bestehen jedoch Interdependenzen, deren negative Folgen für eine verantwortungsbewußt die Zukunft gestaltende Politik derzeit allenthalben beklagt werden.

Die besondere Bedeutung der Sphäre des Ressourcentransfers kann an einem zweiten Beispiel, der Konzeption der Institution des Lebenslaufs, vertiefend erläutert werden. Kohli $(1985 ; 1986)$ hat eindringlich darauf hingewiesen, daß die Institutionalisierung des Lebenslaufes auf der einen Seite zur Verzeitlichung und auf der anderen Seite zur Biographisierung des Lebenslaufs beiträgt. Verzeitlichung ist nun der Aspekt des Lebenslaufs, der für funktional ausdifferenzierte Systeme die Biographie von Individuen in eine handhabbare, bearbeitbare und letztlich benutzbare Form übersetzt. Auf der anderen Seite jedoch ist Biographisierung des Lebensverlaufes diejenige Seite der Institution des Lebensverlaufs, die es gestattet, individuelle Biographieplanungen und individuelle Handlungsmotivationen an die Arbeitsweise funktional ausdifferenzierter Systeme anzubinden. Die Institution des Lebenslaufs verknüpft die Eigenlogik des privaten Lebensbereichs mit der Eigenlogik funktional ausdifferenzierter Systeme. Mit der Institution des Lebenslaufs wird die Verzahnung beider Bereiche gewährleistet. Sie ist somit für beide Handlungssphären eine Außenseite, die jeweils benutzt wird aus der Binnenperspektive des privaten Lebensbereichs und aus der Perspektive sozialer Systeme. Die Institutionalisierung des Le- benslaufs verwandelt individuelle Biographien in berechenbare Lebensverläufe und umgekehrt berechenbare Lebensverläufe in individuell zurechenbare Biographien. Der Lebenslauf als Institution öffnet, weil er ein Bestandteil des Ressourcentransfers ist, biographische Komplexität dem $\mathrm{Zu}$ griff ausdifferenzierter Systeme, wie er auch funktional ausdifferenzierte Systeme dem Zugriff individueller biographischer Handlungsentwürfe öffnet.

Das dritte Beispiel kreist um den Bereich der Wirtschaft, deren Abhängigkeit vom Ressourcentransfer hinlänglich bekannt ist. Insbesondere die sogenannte elektronische Revolution hat nun aber wirtschaftliche Verflechtungen qua Ressourcen erheblich intensiviert. Wenn man etwa den Analysen von Robert Reich (1993) Glauben schenkt, dann liegt in der intelligenten Nutzung und Verknüpfung zugänglicher Möglichkeiten nahezu die einzige Chance für die entwickelten Industriegesellschaften, ihren Anteil an der wirtschaftlichen Wertschöpfung zu halten oder gar noch weiter zu erhöhen. Im klassischen, nicht nur den Marx'schen Analysen zugrundeliegenden Modell, waren Märkte zwar eine wichtige Voraussetzung dafür, daß effizient produziert werden konnte. Über den wirtschaftlichen Erfolg und damit auch die Reproduktion ökonomischer Strukturen entschied aber letztlich der Produktionsprozeß, also die möglichst effiziente und intensive Verknüpfung der Produktionsfaktoren. In hartem Kontrast zu diesem Bild zeichnet Reich ein Bild der modernen Weltwirtschaft, bei dem die intelligente Innovation im Sinne einer Verknüpfung zugänglichen Wissens mit Anwendungsbezügen und neuen Produktideen allein einen fortdauernden Reichtum der Industriegesellschaften garantiert (Reich 1993; $93 \mathrm{ff}$. und 189 ff.). Der klassische industrielle Produktionsprozeß, nach landläufiger Auffassung der eigentliche Bereich des Wirtschaftens, hat dagegen nur noch marginale Bedeutung. Entscheidend für den wirtschaftlichen Erfolg wird es dagegen sein, $d a \beta$ Menschen aus der Vielzahl zugänglicher Ressourcen immer neue und immer intelligentere Verknüpfungen entwickeln (Reich 1993; 207 ff.; 252 ff.).

\section{Zusammenfassung und Schluß}

Die Theorie funktionaler Differenzierung beherrscht das gegenwärtige soziologische Denken zum Themenkreis gesellschaftliche Modernisierung. An ihr entzündet sich insofern jedoch ein zunehmendes Unbehagen als sie eindeutig zu kurz 
greift, wenn man sie als Gesamttheorie gesellschaftlicher Modernisierung interpretiert. Deswegen häufen sich auch die Versuche, ihren Bedeutungsgehalt zu präzisieren und zugleich vernachlässigte Aspekte zu thematisieren. Unseres Erachtens gehen diese Versuche jedoch noch nicht weit genug. Deswegen wird in diesem Beitrag eine Ergänzung des Theorems funktionaler Differenzierung um zwei weitere Themenkomplexe vorgeschlagen, die in dem soziologischen und sozialphilosophischen Denken zum Thema gesellschaftliche Modernisierung immer schon eine wichtige Bedeutung hatten. Bisher wurden sie jedoch eher als Alternativen gegenüber der Theorie funktionaler Differenzierung verstanden. In diesem Beitrag wird nun der Versuch gemacht, die Theorie funktionaler Differenzierung mit dem Aspekt der Marktvergesellschaftung zu verzahnen. Das soziologische Verständnis gesellschaftlicher Modernisierung soll dadurch weiterentwickelt werden, daß das Theorem funktionaler Differenzierung mit dem im Liberalismus und Marxismus betonten Modell der zunehmenden Vergesellschaftung über den Markt zusammengeführt wird. Wenn man bei beiden Theoriesträngen gewisse Akzentverschiebungen vornimmt, dann, so unsere Behauptung, lassen sie sich zu einer Theorie gesellschaftlicher Modernisierung zusammenfügen.

Als gesellschaftliche Modernisierung wird dabei jener historische Prozeß verstanden, bei dem sich segmentär differenzierte Gesellschaften in funktional differenzierte, der Tendenz nach globale Wissens- und Arbeitssysteme verwandelt haben. Dieser Prozeß umfaßt drei interdependente, jedoch eigenlogische Entwicklungen. Ein Rückgriff auf Webers Überlegungen zur Ausdifferenzierung von Berufen, also auf einen Prozeß, der historisch am Anfang dieser Umstellung sozialer Systeme steht, vermag dies plausibel zu macht. Denn die Ausdifferenzierung „früher" Berufe wie Priester oder Schamane macht bereits deutlich, daß berufliche Spezialisierungsprozesse, die sich in der Theorie funktionaler Differenzierung zutreffend beschreiben lassen, zumindest zwei weitere soziale Arrangements voraussetzen. Einmal müssen räumlich expandierende Außenbeziehungen entwickelt werden, bei denen es zu einem Austausch zwischen beruflichen Leistungen und Versorgungsleistungen kommt. An diesen Prozeß $B$ ist, wie sich leicht sehen läßt, die Reproduktion spezialisierter Funktionssysteme gebunden. Zum zweiten müssen bereits die Inhaber "früher" Berufspositionen unterscheiden können zwischen ihrer Berufsrolle und ihren persönlichen Reproduktions- bedürfnissen. Sie lösen sich also in zweierlei Hinsicht, als Positionsinhaber wie als konkrete Person, aus den intensiven Gruppenprozessen sozialer Gemeinschaften heraus. Dieser Individualisierungsprozeß erklärt sowohl das für moderne Gesellschaften unverzichtbare Selektionspotential wie auch ihr notwendig individualisiertes Motivationspotential. Damit wird einmal ein direkter Zusammenhang zwischen der Entwicklung personenspezifischer Fähigkeiten, Neigungen, Interessen, Lebensgeschichten und der Nachfrage nach gesellschaftlichen Ressourcen bzw. dem Zugang zu spezialisierten Wissens- und Arbeitssystemen hergestellt. In der Wechselwirkung zwischen Eigenschaften der konkreten Person und zugänglichen Ressourcen entwickeln sich nicht nur die Individuen, sondern fließt der Gesellschaft energetisches Potential zu, das sowohl selektiv wirksam wird wie auch Handlungen und Arbeitsaktivitäten antreibt und verdichtet. Darüber hinaus muß der individualisierte Mensch auch als disziplinierter und zivilisierter Akteur auftreten, um den Prozeß funktionaler Differenzierung wie auch den Ressourcentransfer in Gang zu halten und weiter zu entwickeln.

Ressourcen sind Produkte menschlicher Arbeit. Sie sind Güter, Dienstleistungen oder Informationen, die auf eine verallgemeinerte Nachfrage hin codiert sind; Gebrauchswerte im Marx'schen Sinne. Darüber hinaus ist für den Ressourcentransfer konstitutiv, daß die Ressourcen der Möglichkeit nach universell, vor allem von der räumlichen Situierung unabhängig zugänglich sind. Dieser $\mathrm{Zu}$ gänglichmachung dient die Entwicklung der Infrastruktur: Verkehrs- und Kommunikationssysteme, Massenmedien, Energie- und Wasserversorgung usw. Diese Tendenz einer allseitigen Ressourcenversorgung wird allerdings immer noch durch Ungleichverteilungen der Zugangsrechte (ungleiche Einkommens- und Vermögensverteilung, Diskriminierung nach Rasse, Geschlecht usw.) behindert und gebremst. Das Zentralthema dieses Entwicklungsstranges gesellschaftlicher Modernisierung ist deswegen auch die Erschließung und Vernetzung der Welt ${ }^{22}$ und die gegenseitige Zugänglich-

22 Mit dieser Feststellung ist zugleich der Unterschied zu Luhmanns (1971) Überlegungen zur Weltgesellschaft markiert. Luhmann setzt in seiner Konzeption der Weltgesellschaft diese als bereits realisiert voraus, weil soziale Systeme per definitionem alle kommunikativen Handlungen umfassen. Diese Vorentscheidung macht es jedoch unmöglich, den durch Ungleichzeitigkeiten und regionale Disparitäten gekennzeichneten Prozeß der Entstehung einer Weltgesellschaft konzeptionell erfassen zu können. 
machung von Ressourcen, die durch Ungleichverteilungen der Zugangsrechte und die Diskriminierung sozialer Großgruppen immer wieder blokkiert wird.

Innerhalb dieses Rahmens kann sich der Prozeß funktionaler Differenzierung entfalten. Funktionale Differenzierung wird als ,Fortschrittsmechanismus" begriffen, der die dauerhafte Konzentration von Personen auf thematisch ausdifferenzierte Wissens- und Arbeitsfelder gewährleisten soll. In Folge dieses Differenzierungsprozesses entwickeln sich Themen- und funktionsspezifische Eigenlogiken, die zu einer Dezentrierung der sozialen Welt, zu ihrer funktionsspezifischen, jedoch räumlich indifferenten Differenzierung führen. An die Entwicklung spezialisierten Wissens ist auch das für Gesellschaften immer konstitutive Element einer überindividuellen, gesellschaftlichen Autorität geknüpft. Sie wird immer polyzentrischer und damit auch unübersichtlicher und in ihrer Reichweite beschränkter. Als „soziales Band“ bleibt sie zumindest innerhalb der ausdifferenzierten Systeme erhalten. Im Rahmen der Theorie funktionaler Differenzierung lassen sich vor allem die von Durkheim verfolgten Fragekomplexe, Arbeitsteilung und Systemdifferenzierung, verfolgen. Was folgt aus diesen Überlegungen für das Problem gesellschaftlicher Integration? Zunächst einmal ist vorstellbar, daß entwickelte Industriegesellschaften zwar nicht ganz ohne normative Regulative auskommen, aber doch mit wesentlich weniger Moralund Wertvorstellungen. Zudem konzentrieren sie sich immer stärker auf die Rahmenbedingungen des Ressourcentransfers und auf die Verteilung der $\mathrm{Zu}$ gangsrechte zu Ressourcen. Alle Diagnosen, die sich in der Tradition des Anomieverdachtes bewegen, verkennen die mit der Durchsetzung der Ressourcenversorgung einhergehende Umstellung selektiver Prozesse auf persönliche oder systemspezifische Interessen. In diesem Beitrag wird dagegen ein anders gelagertes Integrationsproblem moderner Gesellschaften betont, das im wesentlichen eines der beschränkten und ungleich verteilten $\mathrm{Zu}$ gangsrechte zu einem immer stärker universalisierten System des Ressorcentransfers ist.

\section{Literatur}

Alexander, J. C./Giesen, B., 1987: From reduction to linkage: The long view of the micro-macro debate. S. $1-42$ in: Jeffrey C. Alexander u.a. (Hrsg.): The micro-macro link. Berkley-Los Angeles-London: University of California Press.
Allerbeck, K. R./Hoag, W. J., 1989: „Utopia is Around the Corner". Computerdiffusion in den USA als soziale Bewegung. Zeitschrift für Soziologie, Jg.18, H.1:35-53.

Beck, U., 1980: Die Vertreibung aus dem Elfenbeinturm. Anwendung soziologischen Wissens als soziale Konfliktsteuerung. Soziale Welt, Jg.31: 415-441.

Berger, J., 1986: Gibt es ein nachmodernes Gesellschaftsstadium? Marxismus und Modernisierungstheorie im Widerstreit. S. 79-96 in: ders. (Hrsg.): Die Moderne Kontinuitäten und Zäsuren (Sonderband 4; Soziale Welt) Göttingen: Schwartz \& Co.

Brock, D., 1991: Der schwierige Weg in die Moderne. Umwälzungen in der Lebensführung der deutschen Arbeiter zwischen 1850 und 1980. Frankfurt/Main; New York: Campus.

Brock, D., 1993: Wiederkehr der Klassen? Über Mechanismen der Integration und der Ausgrenzung in entwickelten Industriegesellschaften. Soziale Welt, Jg.44, H.2: 177-198.

Castoriadis, C., 1984: Gesellschaft als imaginäre Institution. Entwurf einer politischen Philosophie. (zuerst 1975). Frankfurt a. Main: Suhrkamp.

Corning, P. A., 1982: Durkheim and Spencer. The British Journal of sociology, Vol.33: 359-382.

Durkheim, E., 1987: Der Selbstmord. (Übersetzt von Sebastian und Hanne Herkommer) 2.Aufl. (zuerst 1897). Frankfurt/Main: Suhrkamp.

Durkheim, E., 1988: Über soziale Arbeitsteilung. Studie über die Organisation höherer Gesellschaften. (Mit einer Einleitung von Niklas Luhmann: Arbeitsteilung und Moral. Durkheims Theorie; mit einen Nachwort von Hans-Peter Müller und Michael Schmid.) 2.Aufl. (zuerst 1893). Frankfurt/Main: Suhrkamp.

Eder, K., 1988: Die Vergesellschaftung der Natur. Studien zur sozialen Evolution der praktischen Vernunft. Frankfurt/Main: Suhrkamp.

Elias, N., 1976: Über den Prozess der Zivilisation. Soziogenetische und psychogenetische Untersuchungen. 2 Bde. (zuerst 1936). Frankfurt/Main: Suhrkamp.

Fabio di, U., 1991: Offener Diskurs und geschlossene Systeme. Das Verhältnis von Individuum und Gesellschaft in argumentations- und systemtheoretischer Perspektive. Berlin: Duncker \& Humblot.

Fourastié, J.. 1954: Die große Hoffnung des zwanzigsten Jahrhunderts, (zuerst 1949). Köln-Deutz: Bund.

Freyer, H., 1955: Theorie des gegenwärtigen Zeitalters. Stuttgart: Deutsche Verlags Anstalt.

Gehlen, A., 1956: Urmensch und Spätkultur. Philosophische Ergebnisse und Aussagen. Bonn: Athenäum.

Giddens, A., 1976: Functionalism: Après la lutte. Social Research, Vol.43: 325-366.

Giddens, A., 1988: Die Konstitution der Gesellschaft. Grundzüge einer Theorie der Strukturierung. (Mit einer Einführung von Hans Joas, Orig.: The Constitution of Society. Outline of the theory of strucuration.) (zuerst 1984). Frankfurt/New York: Campus.

Goffman. E., 1983: Wir alle spielen Theater. Die Selbstdarstellung im Alltag. 4.Aufl. (zuerst 1959). München: Piper. 
Habermas, J., 1981: Theorie des kommunikativen Handelns. 2 Bde. Frankfurt/Main: Suhrkamp.

Heckmann, F./Kröll, F., 1984: Einführung in die Geschichte der Soziologie. Stuttgart: Enke.

Hitzler, R., 1994: Die banale Seite der Macht. S. 280-295 in: Helmuth Berking/Ronald Hitzler/Sighard Neckel (Hrsg.): Politikertypen in Europa. Frankfurt a. Main: Fischer.

Hondrich, K. O., 1992: Wovon wir nichts wissen wollten. Die Zeit, Nr.40 vom 25.09.1992:

Joas, H., 1986: Die unglückliche Ehe von Hermeneutik und Funktionalismus. S. 144-176 in: Axel Honneth/ ders. (Hrsg.): Kommunikatives Handeln. Beiträge zu Jürgen Habermas' „Theorie des kommunikativen Handelns". Frankfurt a. Main: Suhrkamp.

Joas, H., 1990: Die Demokratisierung der Differenzierungsfrage. Die Krise des Fortschrittsglaubens und die Kreativität des kollektiven Handelns. Soziale Welt, Jg.41, H.1: 8-27.

Joas, H., 1992: Die Kreativität des Handelns. Frankfurt a. Main: Suhrkamp.

Kohli, M., 1985: Die Institutionalisierung des Lebenslaufs. Historische Befunde und theoretische Argumente. Kölner Zeitschrift für Soziologie und Sozialpsychologie, Jg.37, H.1: 1-29.

Kohli, M., 1986: Gesellschaftszeit und Lebenszeit. Der Lebenslauf im Strukturwandel der Moderne. S. 183-208 in: Johannes Berger (Hrsg.): Die Moderne - Kontinuitäten und Zäsuren (Soziale Welt: Sonderband 4). Göttingen: Otto Schwartz \& Co.

Kruse, V., 1994: Historisch-soziologische Zeitdiagnosen in Westdeutschland nach 1945. Eduard Heimann, Alfred von Martin, Hans Freyer. Frankfurt a. Main: Suhrkamp.

Künzler, J., 1989: Medien und Gesellschaft. Die Medienkonzepte von Talcott Parsons, Jürgen Habermas und Niklas Luhmann. Stuttgart: Enke.

Landmann M., 1987: Einleitung des Herausgebers. S. 7-29 in: ders. (Hrsg.): Georg Simmel. Das individuelle Gesetz. Philosophische Exkurse. Neuausgabe (zuerst 1968).Frankfurt: Suhrkamp.

Lockwood, D., 1964: Social integration and system integration. S. 244-257 in: G.Z. Rollschan/W. Hirsch (Hg.) (Hrsg.): Explorations in social change. London: .

Luhmann, N., 1971: Die Weltgesellschaft. Archiv für Rechts- und Sozialphilosophie, Jg.57: 1-35.

Luhmann, N., 1975: Evolution und Geschichte, in: ders. (Hrsg.): Soziologische Aufklärung Bd.2. Opladen: Westdeutscher Verlag.

Luhmann, N., 1984: Soziale Systeme. Grundriß einer allgemeinen Theorie. Frankfurt/Main: Suhrkamp.

Luhmann, N., 1988: Arbeitsteilung und Moral. Durkheims Theorie. S. 19-38 in: Emile Durkheim (Hrsg.): Über soziale Arbeitsteilung. Studie über die Organisation höherer Gesellschaften. Frankfurt/Main: Suhrkamp.

Luhmann. N., 1993: Gesellschaftsstruktur und Semantik. Studien zur Wissenssoziologie der modernen Gesellschaft. Bd. 1. Frankfurt/Main: Suhrkamp.

Lyotard, J.-F, 1986: Das postmoderne Wissen. Ein Bericht, (zuerst 1979). Graz/Wien: Edition Passagen.
Marx, K., 1973: Das Kapital. Kritik der politischen Ökonomie. (MEW Bd. 23-25) (zuerst 1867). Berlin (Ost): Dietz.

Marx, K., 1974: Grundrisse der Kritik der politischen Ökonomie. Berlin: Dietz

Mayntz, R., 1993: Große technische Systeme und ihre gesellschaftstheoretische Bedeutung. Kölner Zeitschrift für Soziologie und Sozialpsychologie, Jg.45: 97-108.

Mill, J. S., 1976: Der Utilitarismus. (übersetzt von D. Birnbacher), (zuerst 1863). Stuttgart: Reclam.

Müller, H.-P./Schmid, M., 1988: Arbeitsteilung, Solidarität und Moral. Eine werkgeschichtliche und systematische Einführung in die "Arbeitsteilung" von Emile Durkheim. S. 481-532 in: Emile Durkheim (Hrsg.): Über soziale Arbeitsteilung. Studie über die Organisation höherer Gesellschaften. Frankfurt/Main: Suhrkamp.

Müller, H.-P./Schmid, M. (Hrsg.), 1994: Sozialer Wandel. Modellbildung und theoretische Ansätze. Frankfurt a. Main: Suhrkamp.

Münch, R., 1984: Die Struktur der Moderne. Grundmuster und differentielle Gestaltung des institutionellen Aufbaus der modernen Gesellschaft. Frankfurt/Main: Suhrkamp.

Münch, R., 1988: Theorie des Handelns. Zur Rekonstruktion der Beiträge von Talcott Parsons, Emile Durkheim und Max Weber. 1. Aufl. (zuerst 1982). Frankfurt/ Main: Suhrkamp.

Münch, R., 1993: Die Kultur der Moderne. Band 1: Ihre Grundlagen und ihre Entwicklung in England und Amerika. Band 2: Ihre Entwicklung in Frankreich und Deutschland. Frankfurt/Main: Suhrkamp.

Münch, R., 1994: Zahlung und Achtung. Die Interpenetration von Ökonomie und Moral. Zeitschrift für Soziologie, Jg.23: 388-411.

Parsons, T., 1980: Sozialstruktur und die symbolischen Tauschmedien. (Orig. Social Structure and the Symbolic Media of Interchange.) In: Talcolt Parsons. Zur Theorie der sozialen Interaktionsmedien. (Herausgegeben und eingeleitet von Stefen Jensen.) (zuerst 1975). Opladen: Westdeutscher Verlag.

Reich, R., 1993: Die neue Weltwirtschaft. Berlin: Ullstein.

Schelsky, H., 1973: Zur soziologischen Theorie der Institution. S. 9-26 in: ders. (Hrsg.): Zur Theorie der Institution. Düsseldorf: Bertelsmann.

Schmid, M., 1989: Arbeitsteilung und Solidarität. Eine Untersuchung zu Emile Durkheims Theorie der sozialen Arbeitsteilung. Kölner Zeitschrift für Soziologie und Sozialpsychologie, Jg.41, H.4: 619-643.

Simmel, G., 1983: Schriften zur Soziologie. Eine Auswahl. (Herausgegeben und eingeleitet von Heinz-Jürgen Dahme und Ottheim Rammstedt) Frankfurt a.M.: Suhrkamp.

Simmel, G., 1992: Soziologie. Untersuchungen über die Formen der Vergesellschaftung. (Gesamtausgabe Band 11. Herausgegeben von Otthein Rammstedt) (zuerst 1908). Frankfurt a. Main: Suhrkamp.

Stehr, N., 1994: Arbeit, Eigentum und Wissen. Zur Theorie von Wissensgesellschaften. Frankfurt a. Main: Suhrkamp. 
Touraine, A., 1976: Was nützt die Soziologie? (zuerst 1974). Frankfurt a. Main: Suhrkamp.

Touraine, A., 1988: Modernity and Cultural Specificities. International Social Science Journal, 40: 443-457.

Tyrell, H., 1978: Anfragen an die Theorie der gesellschaftlichen Differenzierung. Zeitschrift für Soziologie, 7. Jg.: 175-193.

Tyrell H., 1985: Emile Durkheim - Das Dilemma der organischen Solidarität. S. 181-250 in: Niklas Luhmann (Hrsg.): Soziale Differenzierung. Zur Geschichte einer Idee. Opladen: Westdeutscher.

Weber, M., 1985: Wirtschaft und Gesellschaft. Grundriss der verstehenden Soziologie. (Besorgt von Johannes
Winckelmann) 5.rev. Aufl. Studienausgabe (zuerst 1922). Tübingen: J.C.B. Mohr (Paul Siebeck).

Weber, M., 1988: Gesammelte Aufsätze zur Religionssoziologie. 3 Bde. (zuerst 1920). Tübingen: J.C.B. Mohr (Paul Siebeck).

Wehling, P., 1992: Die Moderne als Sozialmythos. Zur Kritik sozialwissenschaftlicher Modernisierungstheorien. Frankfurt/Main; New York: Campus.

Willke, H., 1991: Systemtheorie. Eine Einführung in die Grundprobleme der Theorie sozialer Systeme. Stuttgart; New York: G. Fischer. 